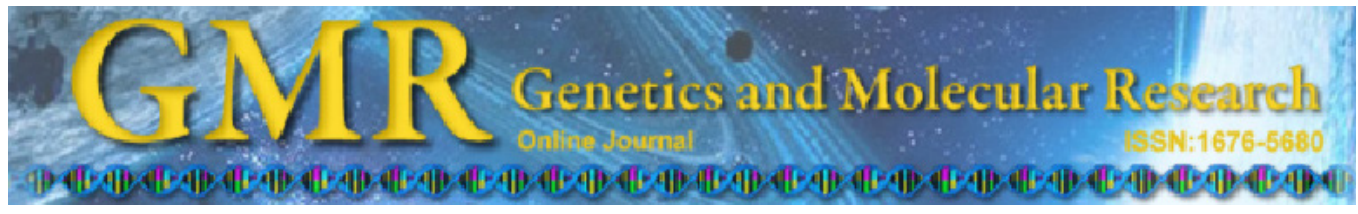

\title{
Genetic diversity of stem cells and their functional impact on the development of neural tube defects in Eastern population of India
}

\author{
A.K. Saxena ${ }^{1}$, S. Pandey ${ }^{2}$ and L.K. Pandey ${ }^{2}$ \\ ${ }^{1}$ Department of Pathology and Lab Medicine, \\ All India Institute of Medical Sciences, Patna, Bihar, India \\ ${ }^{2}$ Department of Obstetrics and Gynecology, Faculty of Medicine, \\ Institute of Medical Sciences, B.H.U., Varanasi, India \\ Corresponding author: A.K. Saxena \\ E-mail: draksaxena1@rediffmail.com
}

Genet. Mol. Res. 12 (3): 2380-2390 (2013)

Received August 1, 2012

Accepted December 7, 2012

Published July 15, 2013

DOI http://dx.doi.org/10.4238/2013.July.15.1

\begin{abstract}
Anencephaly and myelomeningocele are the 2 most common forms of neural tube defects (NTDs). During embryogenesis large numbers of extrinsic and intrinsic factors are responsible for the closing of the neural tube. "Stem cells" maintain the pluripotency during differentiation of 3 germ layers, including the neural ectoderm. We examined the role of Oct4, Nanog3, and Sox 2 genes in the etiopathology of NTDs in an eastern Indian population using PCRbased DNA analysis. The highest frequency $(16 \%)$ of complete loss of the Sox 2 gene was found in NTDs. The highest frequency $(48 \%)$ of overexpression (upregulation) was found for Nanog3, while $40 \%$ was observed for Oct4 and Sox2. The odds ratio for cases versus controls was from 0.132 at $95 \%$ confidence interval $=0.005-1.298$ for Nanog3 to $2.316(0.424-13.812)$ for Oct4. The highest frequency (77\%) of overexpression for Nanog3 and Sox 2 was observed in encephalocele and anencephalic patients, while in the comparison of regional variation, i.e., cephalic to caudal regions of NTDs, the highest
\end{abstract}


frequency of downregulation (regression) of Nanog3 and Sox2 was found in lumbosacral myelomeningocele patients. However, cervical myelomeningocele patients had the highest frequency of overexpression in all 3 genes, suggesting that the mutational spectra of stem cells influence the cells of the neural crest in NTDs.

Key words: Stem cell; Oct4; Nanog3; Sox2; Neural tube defects

\section{INTRODUCTION}

Since the discovery of stem cell marker in 2003, special attention has been given in how to maintain the pluripotency for the development of germ layers including neuronal proliferation (Seller, 1990; Eric et al., 2005). Embryonic stem cells are derived from the inner cell mass of the developing blastocyst and are capable of differentiate into a variety of specific cell lineages, i.e., ectoderm, mesoderm, and endoderm, to form a hollow dorsally placed neural tube - a derivative of the neuroectoderm. In vertebrate embryos, after neural tube closure, the neural crest cells (NCC) detach from the ectoderm at the periphery region to multiply and infiltrate in mesoderm to form central nervous system (Tada and Tada, 2001). During differentiation of the central nervous system, NCC are the consequence of a combination of both extrinsic and intrinsic factors that arise from the embryonic niche. These factors later characterized as "stem cell markers" associated with transcriptional factor maintaining pluripotency during preimplantation of embryo. The original progenitor cells disappear along with their birthplace as the neural tube closes and matures. The NCC generally form neurons, glial cells of the peripheral nervous system, pigment, and endocrine cells. In cephalic region they give rise to tendons, cartilage, bone, dermis, vascular smooth muscle, and adipocytes (Rosner et al., 1990; Partington and McLone, 1995; Couly et al., 2002). However, the dorsal root ganglia, hair follicle, tooth, and even the bone marrow are the derivatives of neural crest-derived stem cells (Le Lievre and Le Douarin, 1975; Nichols et al., 1998; Kruger et al., 2002; Fernandes et al., 2004; Sieber-Blum et al., 2004; Li et al., 2007; Motohashi et al., 2007; Takashima et al., 2007).

Birth defects are the combination of both genetic and epigenetic factors (Finnell et al., 2002; Cabrera et al., 2002; Detrait et al., 2005; Beaudin and Stover, 2009). In a developing country such as India, neural tube defects (NTDs) are in central nervous system including brain and spinal cord, where neural tube fails to close during early embryogenesis. Anencephaly and myelomeningocele (MMC) are the most common forms of NTDs and a large number of genetic variants in a single gene vary in population between different racial/ethnic groups. Earlier studies clearly reveal that periconceptional folic acid supplementation may reduce risk factor up to 75\% (Anonymous, 1991; Van der Put et al., 1995; Botto and Yang, 2000). The incidence of NTDs is highly variable (0.2-3.5 per 1000 births) depending on racial, ethnic, and geographical aspects. MMC is the most severe form of spina bifida and carriers have difficult to survive because of dysplastic spinal cord with lack of neuronal function. In MMC, both meninges and the spinal cord protrude through a gap in the vertebral column and the lesion is not covered by the skin. These anomalies can occur at any point along with the developing neural tube, although lumbosacral lesions are the most common form of NTDs (Hunter et al., 1996). Most children with MMC survive after surgical intervention with lifelong disabilities (Abzhanov et al., 2003). NTDs include MMC (spina bifida aperta), in which the brain remains 
open and the skull vault fails to develop. Babies with spina bifida have little chance to survive postnatally, but often suffer neurological deficit below the level of the lesion and demonstrate associated problems such as hydrocephalus.

The etiologies of NTDs are exceedingly complex due to interaction of extrinsic or intrinsic factors such as stem cell during organogenesis. Considerable interest has been generated to determine how genetic components of stem cell diversity may be used as a "marker" for regenerative source of medicine. In human, Oct4 and Sox 2 are the prime stem cell markers required for cell proliferation to maintain pluripotency. Similarly, Nanog3, a newly identified protein, acts as a transcription factor to maintain pluripotency in embryonic stem cell (Pesce and Scholer, 2001; Mitsui et al., 2003; Chambers and Smith, 2004; Levasseur et al., 2008). The over-expression of Sox 2 in combination with Oct4 play an imperative role in carcinogenesis (Henderson et al., 2002; Pardal et al., 2003) but their functional role in NTDs has not been defined in literature.

However, genetic diversity of stem cells in NTDs and how to maintain pluripotency still require a comprehensive study including sequential use of stem cell markers - Oct 4, Nanog3, and Sox2. Our efforts have been to gain new insight into the understand genetic and epigenetic factors regulating neural tube folding during neurogenesis. Hence, our aim was to reveal genetic components of stem cell identification, their characterization, and to correlate them in different types of clinically diagnosed NTDs with the ultimate goal of create a "regenerative source" for stem cell therapy in modern medicine.

\section{MATERIAL AND METHODS}

Blood samples $(1.0 \mathrm{~mL})$ were collected from clinically diagnosed NTD $(\mathrm{N}=75)$ outpatients in the hospital in different age groups (less than 1 week to 3 months) after written consent from patients/guardians and equal number of age- and gender-matched $(\mathrm{N}=75)$ controls. The criteria for inclusion of an individual were based on clinically diagnosed NTDs. The study was approved by the Ethics Committee of the Institute. Genomic DNA was isolated from whole blood using a Bioner kit (Korea) and samples were kept at $-20^{\circ} \mathrm{C}$ until further analysis.

In the present study of NTDs, cases were randomly selected for the characterization of stem cell markers namely Oct4, Nanog3, and Sox2 using specific upstream/downstream amplicons (primers) as detailed in Table 1.

\begin{tabular}{|c|c|c|c|c|}
\hline Stem cell markers & Sequences (forward and reverse) & Length (bp) & Annealing temperature & References \\
\hline Oct4 & F: 5'-GACCATCTGCCGCTTTGAG-3' & 577 & $60^{\circ} \mathrm{C} / 1 \mathrm{~min}$ & Henderson et al., 2002 \\
\hline Oct4 & R: 5'-CCCCCTGTCCCCCATTCCTA-3' & & & \\
\hline Nanog3 & F: 5'-CTGTGATTTGTGGGCCTG AA-3' & 151 & $56^{\circ} \mathrm{C} / 30 \mathrm{~s}$ & Nettersheim et al., 2011 \\
\hline Nanog3 & R: 5'-TGTTTGCCTTTGGGACTGGT-3' & & & \\
\hline Sox 2 & F: 5'-GGCAGCTACAGCATGATGC-3' & 236 & $60^{\circ} \mathrm{C} / 30 \mathrm{~s}$ & Bhatia et al., 2011 \\
\hline Sox2 & R: 5'-TCGGACTTGACCACCGAAC-3' & & & \\
\hline
\end{tabular}

We developed 3 different PCR-specific strategies for the identification and characterization of stem cells namely Oct4, Nanog3, and Sox2 gene markers using forward and reverse 
primers in a total volume of $25 \mu \mathrm{L}$ containing 50-100 ng DNA, 20 pmol of each primer, 200 $\mu \mathrm{M}$ of each dNTP with Taq buffer (10 mM Tris-HCl, $\mathrm{pH} 8.3,50 \mathrm{mM} \mathrm{KCl}), 3.0 \mathrm{mM} \mathrm{MgCl}$, and $3 \mathrm{U}$ Taq polymerase (New England Biolabs). Cycling conditions were 1 min for Oct4 (577 bp), $4 \mathrm{~min}$ for Nanog 3 , and $2 \mathrm{~min}$ for Sox 2 at $94^{\circ} \mathrm{C}$ for initial denaturation, $60^{\circ} \mathrm{C} / 1 \mathrm{~min}$, $56^{\circ} \mathrm{C} / 30 \mathrm{~s}$ and $60^{\circ} \mathrm{C} / 30 \mathrm{~s}$ of annealing for Oct 4 , Nanog3, and Sox 2 , respectively, followed by a final extension of 35 cycles at $72^{\circ} \mathrm{C}$ for $5 \mathrm{~min}$ for all 3 stem cell markers used. PCR products were separated on $1.5 \%$ agarose gel, stained with ethedium bromide and bands were visualized on the Gel Doc system (SR Biosystem).

Statistical analysis was carried out using the $\chi^{2}$ test for comparison of controls and NTD patients to determine the level of significance. The odds ratio (OD) at $95 \%$ confidence interval $(95 \% \mathrm{CI})$ were calculated to determine the risk factor between NTD cases, NTD mothers, and their respective controls.

\section{RESULTS}

\section{Evaluation of genetic diversity of stem cell in NTDs}

In human, stem cell markers were used to screen mutational spectra in NTDs and their optimal condition for amplification of DNA-coding region of exon having different length of the primers with annealing temperature used for the exploration of Oct4, Nanog3, and Sox 2 as documented in Table 1. Our findings were quite convincing regarding stem cell regulation in NTDs. The observations were based on the appearance of bands and their signal intensity help to characterize specific amplicons for overexpression (upregulation), regression (downregulation), and complete disappearance of band (null) in NTD cases, when compared to the same in controls. The findings of this study were repeated 3 times to confirm the genetic diversity of stem cell as documented in Table 2.

\begin{tabular}{|c|c|c|c|c|}
\hline \multirow[t]{2}{*}{ Types of stem cell markers } & $\underline{\text { Case }}$ & Control & \multirow[t]{2}{*}{ OR $(95 \% \mathrm{CI})$ cases $v s$ controls } & \multirow[t]{2}{*}{$\mathrm{P}$} \\
\hline & \multicolumn{2}{|c|}{ Frequency (\%) } & & \\
\hline \multicolumn{5}{|l|}{ Oct4 } \\
\hline Upregulation & 40 & 20 & $2.667(0.644-11.495)$ & 0.123 \\
\hline Downregulation & 8 & 12 & $2.316(0.424-13.812)$ & 0.269 \\
\hline Null/Absent & 4 & 4 & $0.219(0.009-2.397)$ & 0.157 \\
\hline \multicolumn{5}{|l|}{ Nanog3 } \\
\hline Upregulation & 48 & 36 & $1.641(0.457-5.967)$ & 0.390 \\
\hline Downregulation & 24 & 28 & $0.132(0.005-1.298)$ & 0.747 \\
\hline Null/Absent & 4 & 24 & $0.0812(0.191-3.416)$ & $0.042 *$ \\
\hline \multicolumn{5}{|c|}{ 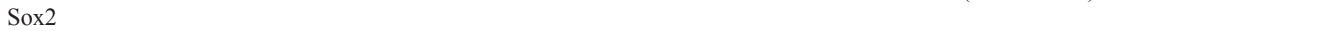 } \\
\hline Upregulation & 40 & 20 & $2.667(0.644-11.495)$ & 0.123 \\
\hline Downregulation & 24 & 40 & $0.638(0.066-5.396)$ & 0.637 \\
\hline Null/Absent & 4 & 16 & $1.000(0.025-39.336)$ & 1.000 \\
\hline
\end{tabular}

*Statistical analysis showing significant differences at $\mathrm{P}<0.05$ using the chi-square test.

\section{Oct4 gene regulation in NTD cases and controls}

The overexpression (upregulation) and regression (downregulation) of Oct4 gene 
(577 bp) in NTD cases and controls are shown in Figure 1A and B. Apparently Figure 2 shows a significant decreasing trend of Oct4 gene mutation whereas the highest frequencies were observed in upregulation (40\%) as shown in Figure 2A, downregulation (8\%), and complete disappearance (4\%) in Figure 2B and C, respectively, between cases and controls. OR and $95 \% \mathrm{CI}$ also showed the highest value of overexpression (upregulation): $\mathrm{OR}=2.667$ (95\% CI 0.644-11.495) with $\mathrm{P}=0.123$.

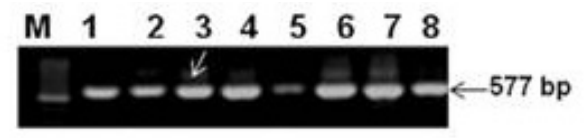

A

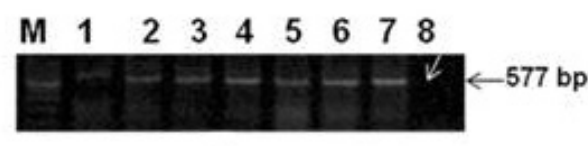

B

Figure 1. A. Representative photograph of amplified product of the Oct4 gene consisting of a 577-bp band analyzed on $1.5 \%$ agarose gel stained with ethidium bromide showing downregulation (lane 5) and upregulation (overexpression) in lanes 3, 4, 6, 7, and 8 in neural tube defect cases. Lanes 1 and $2=$ normal expression. B. Controls showing equal expression of the Oct 4 gene, and lane 8 showing complete deletion of the amplified product. Lane $M$ $=$ molecular marker; lanes 1 and $2=$ downregulation.

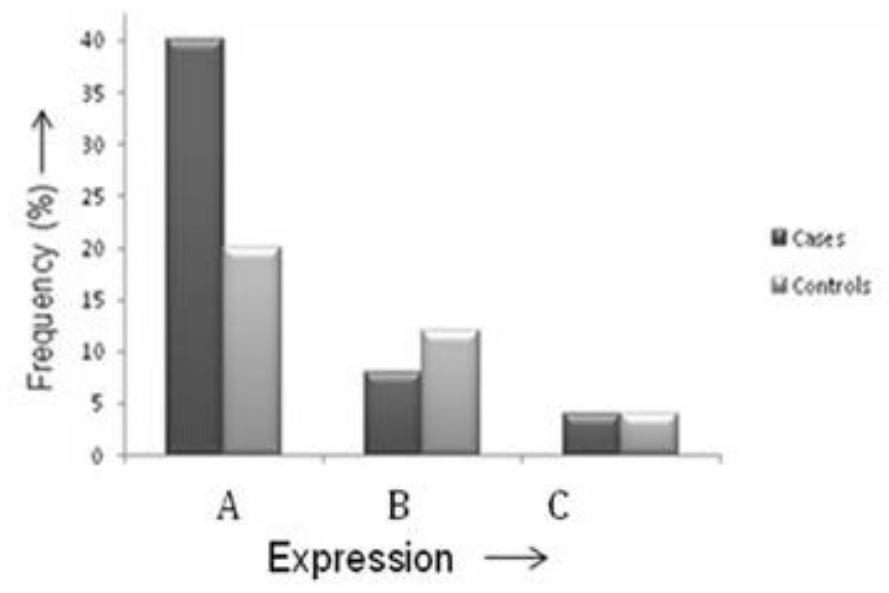

Figure 2. Bar diagram showing the decreasing trend of frequency (\%) of the stem cell Oct4 marker gene mutation upregulation (A), downregulation (B), and complete disapperance of band (C) in neural tube defect cases (black columns) in relation to controls (gray columns).

\section{Nanog3 gene regulation in NTD cases}

Similarly, the overexpression (upregulation), downregulation, and complete disappearance were evaluated for Nanog3 (151 bp) as depicted in Figure 3A and B. The variable frequency of upregulation (48\%), downregulation (24\%), and complete disappearance (4\%) in Nanog3 were observed (Figure 4), which apparently showed significant decreasing trend of gene mutation in NTD cases. OR and 95\%CI showed significant differences $(\mathrm{P}<0.05)$ using the chi-square test for complete disappearance (null): $\mathrm{OR}=0.0812(0.191-3.416)$ with $\mathrm{P}=$ 0.042 between cases and controls. 


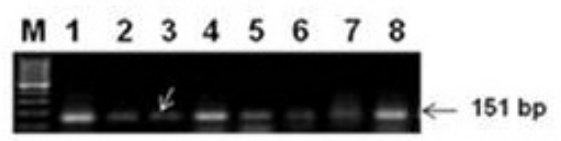

A

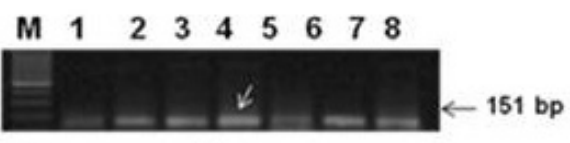

B

Figure 3. A. PCR product of the Nanog3 gene consisting of $151 \mathrm{bp}$ showing overexpression in lanes 1,4 , and 8 , and downregulation in lanes 2, 3, 5, 6, and 7. B. Controls showing downregulation in lanes 1 and 6 . Lane $M=$ molecular marker.

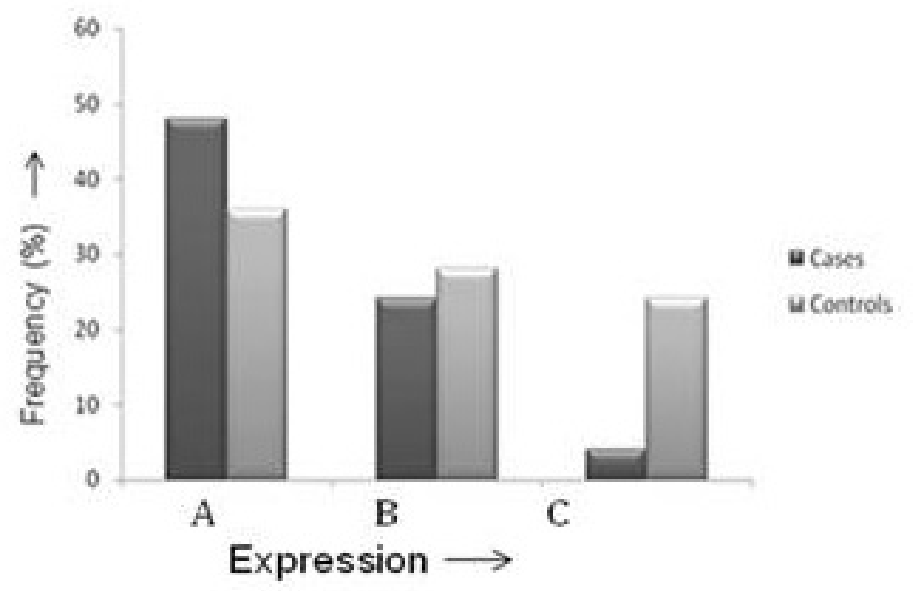

Figure 4. Bar diagram showing the decreasing trend of frequency (\%) of the stem cell Nanog3 marker gene mutation upregulation (A), downregulation (B), and complete disapperance (C) in neural tube defect cases (black columns) in relation to controls (gray columns).

\section{Sox2 gene regulation in NTDs cases and controls}

The overexpression (upregulation), downregulation, and complete disappearance of Sox2 (236 bp) amplicon was observed between NTD cases and controls as shown in Figure 5A and B. The Sox 2 gene mutation showed decreasing trend as depicted in Figure 6, whereas the highest frequency was observed in upregulation (40\%), downregulation (24\%) and complete disappearance (4\%) between cases and controls, but when statistically analyzed showed lack of significant differences. OR again showed the highest value of overexpression (upregulation) where $\mathrm{OR}=2.667(0.644-11.495)$ with $\mathrm{P}=0.123$ observed between cases and controls.

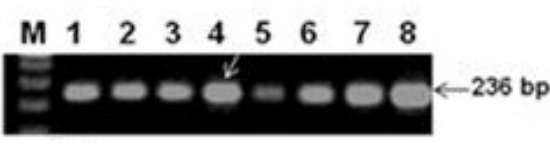

A

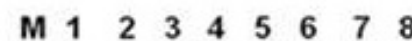

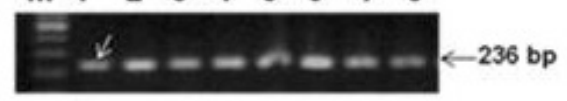

B

Figure 5. A. Sox 2 gene mutation ( $236 \mathrm{bp}$ ) showing upregulation (lanes 4,7 , and 8 ) and downregulation (lanes 3 and 5) in neural tube defect cases. Lanes 1, 2 and $6=$ normal expression of the Sox 2 gene in NTD cases. B. Controls showing similar expression. Lane $M=$ molecular marker. 


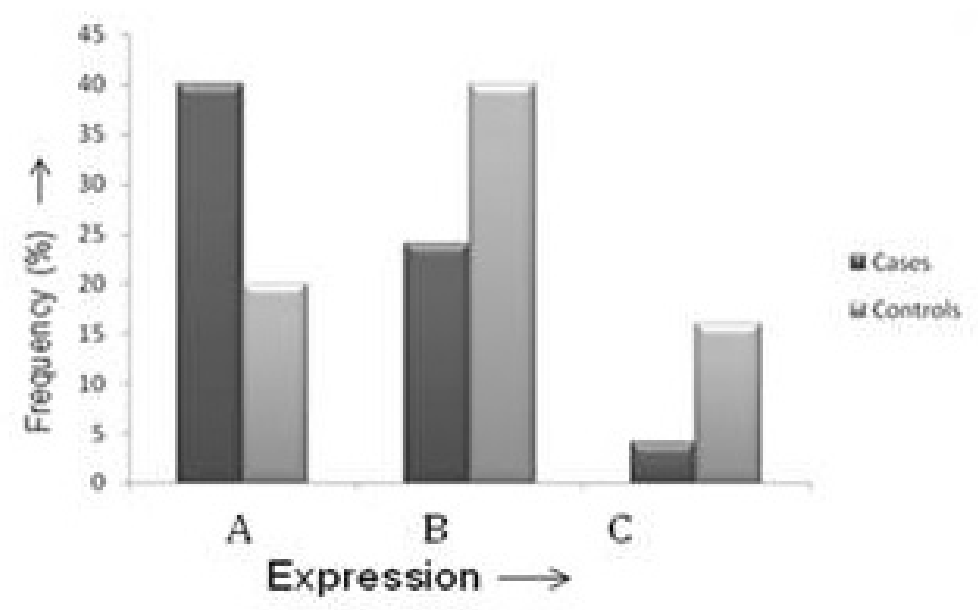

Figure 6. Bar diagram showing the decreasing trend of frequency (\%) of the stem cell Sox 2 marker gene mutation upregulation (A), downregulation (B), and complete disapperance (C) in neural tube defect cases (black columns) in relation to controls (gray columns).

\section{Genetic diversity of stem cell in clinically diagnosed NTDs}

The data were further analyzed to evaluate the genetic diversity of stem cells on the basis of severity of diseases in cases and to find out which stem cell marker promotes genetic susceptibility towards development of NTDs. The finding reveals that encephalocele was the most severe form of NTDs showing very high frequency in overexpression of Nanog3 and Sox2. The decreasing trend was observed when findings were analyzed on the basis of affected region or specific site of involvement, i.e., from caudal region to cephalic end including thoracic and lumbosacral region. The cervical region showed the highest incidence of overexpression in Oct4 and Nanog3, while minimum for Sox2. Similarly, the prevalence (33.3\%) of downregulation (regression) of Nanog3 and Sox 2 were observed in encephalocele and lumbosacral MMC cases of NTDs. Lumbosacral MMC showed a variable trend in overexpression of all 3 stem cell markers, i.e., maximum of $66.6 \%$ in Nanog $3,45.0 \%$ in Sox 2 , and $33.33 \%$ in Oct4, as documented in Table 3.

Table 3. Genetic heterogeneity of stem cells in clinically diagnosed neural tube defect (NTD) patients on the basis of their severity.

\begin{tabular}{|c|c|c|c|c|c|c|}
\hline \multirow[t]{2}{*}{ Types of NTDs } & \multicolumn{2}{|c|}{ Oct4 (\%) } & \multicolumn{2}{|c|}{ Nanog3 (\%) } & \multicolumn{2}{|c|}{ Sox $2(\%)$} \\
\hline & Upregulation & Downregulation & Upregulation & Downregulation & Upregulation & Downregulation \\
\hline Anencephaly & 66.6 & 33.3 & 66.6 & 33.3 & 66.6 & 33.3 \\
\hline Encephalocele & 66.6 & 33.3 & 77.7 & 23.3 & 77.7 & 23.3 \\
\hline CMMC & 100.0 & 00.0 & 100.0 & 00.0 & 50.0 & 50.0 \\
\hline LMMC & 33.3 & 66.6 & 66.6 & 33.3 & 45.0 & 55.0 \\
\hline TMMC & 66.6 & 33.3 & 33.3 & 66.6 & 33.3 & 66.6 \\
\hline Hydrocephalous & 100.0 & 00.0 & 100.0 & 00.0 & 100.0 & 00.0 \\
\hline Open NTDs & 100.0 & 00.0 & 25.0 & 75.0 & 25.0 & 75.0 \\
\hline
\end{tabular}

CMMC, LMMC, and TMMC = cervical, lumbosacral, and thoracic myelomeningocele, respectively. 


\section{DISCUSSION}

Genetic diversity of stem cell becomes quite interesting because variable expression in different types of clinically diagnosed NTDs has not been documented earlier. The specification of cell lineages in the developing brain is thought to be regulated by either extrinsic or intrinsic factors. In human, Oct4, Nanog3, and Sox2 genes are the key regulators of transcriptional activity during organogenesis (Motohashi et al., 2007). It is evident that maintaining pluripotency by sequential changes in the expression of stem cell gene, i.e., overexpression or downregulation or complete disappearance (null) perhaps lead to modify the whole sequence of differentiation of neural ectoderm, where the onset of neural tube defects fail to close due to temporary or permanent gene mutation. This diversity of stem cell gene regulation in NTDs is a unique phenomenon of pluripotency during development of central nervous system. Anencephaly and encephalocele are recognized as being the highest degree of severity leading to lethal condition of NTDs and affected individuals die in utero showing high incidence of upregulation (overexpression) in Nanog3 and Sox 2 gene mutation, suggesting either fail to maintain pluripotency during early embryonic development or fail to neural crest responsible for folding of neural tube. The present observations of overexpression (upregulation) in Oct4 have been observed in NTDs with lack of significant difference between cases and controls, suggesting maintenance of autopluripotency in early embryonic development during formation of neural ectoderm either independently or synchronized with other stem cell gene such as Nanog3/Sox2. The Oct 4 gene is required during early embryonic development - it is a member of mammalian POU family of transcriptional factor genes and its depletion or deletion (absent) in vivo/in vitro results in obstruction of differentiated extra-embryonic trophoectoderm (Finnell et al., 2002; Cabrera et al., 2002; Beaudin and Stover, 2009). This implies that Oct4 acts as an important key regulatory molecule in deciding the fate of embryo during organogenesis as also evident from the present study. However, increasing expression of Oct4 in embryonic stem cells leads to differentiate extra-embryonic endoderm. Oct4 plays a crucial role during embryonic development, maintenance of both self-renewal ability and pluripotency of embryonic stem cells. Maternally derived Oct 4 expression in fertilized oocytes allows correct formation of embryonic and extra-embryonic tissues such as neural tissue during differentiation of central nervous system. This divergent role of Oct4 suggests that Oct 4 transcriptionally regulates those genes involved in coordinating multiple cellular functions (Deb-Rinker et al., 2005; Levasseur et al., 2008).

During implantation Nanog3 is critically required for the maintenance of pluripotency. Null mutation of the Oct4 and Sox 2 genes results in lethality or severity in early embryonic life as also observed in the present study in cases of anencephaly and encephalocele. Oct4 and Sox 2 molecules are required for transcriptional regulation of Nanog3 gene expression. During embryogenesis both Oct4 and Nanog3 are initially required for the maintenance of pluripotency. Hence, Nanog-null embryos seem to be able to initially give rise to pluripotent cells, but these cells then immediately differentiate into the extra-embryonic endoderm lineage. During gastrulation, cells start to migrate at the time of primitive streak formation showing downregulation of Nanog3. Similar observations were also observed in encephalocele and thoracic MMC cases (Hart et al., 2004). Although, Oct4 and Nanog3 act as key markers to regulate stem cell differentiation in NTDs, due to a high incidence of overexpression they may be associated with the severity of the disease. However, upregula- 
tion of Nanog3 with Oct4 and Sox 2 is associated with hypermethylation during neuronal differentiation in human (Deb-Rinker et al., 2005). Thus, Nanog seems to play a crucial role in decide the fate between early and late blastocyst and could be used as an important stem cell marker for self-renewal leading to maintenance of undifferentiated extra-embryonic endoderm.

The pluripotency of neural stem cells has been poorly described about the regulation of the expression of Oct4, Nanog3, and Sox2, critical transcription factors in early embryonic life (Seller, 1990). The mimicry of Sox 2 gene regulation (down/overexpression) has been well documented in cancer patients (Rosner et al., 1990; Couly et al., 2002). However, similar findings for the Sox 2 gene have been reported in case of encephalocele and hydrocephalus. Since overexpression could be due to unbalanced genomic rearrangements or transcription factors at the Sox 2 locus, we could not rule out that Sox 2 promoter represents an enrichment of the positive transcription mark $\mathrm{H} 3 \mathrm{k} 4 \mathrm{me} 3$, which required further experiments to test this hypothesis (pluripotency) that Sox2 is needed to maintain neural stem cell identity in the developing brain. Constitutive expression of Sox 2 inhibits neuronal differentiation and preserved neural progenitor characteristics, whereas, inhibition of Sox2 lead to an early onset of neuronal differentiation (Bhatia et al., 2011). In the adult brain, Sox 2 expression is retained in the neural stem cell compartment. Perhaps this study will help to evaluate the sequential changes in the expression of neuronal cell differentiation and stem cell genetic diversity in clinically classified NTDs. However, Oct4, Nanog3, and Sox 2 are required to maintain pluripotency for neuronal differentiation by up- or downregulation in cerebellum of adult brain and even in medulloblastoma of a child (Nettersheim et al., 2011). Finally, it is clear from the present study that these stem cell markers are key determinants of self-renewal including transcriptional regulation early embryogenesis, chromatin modification, genomic imprinting, and maintenance of phenotypes.

\section{CONCLUSION}

In human, the exact cause of NTD is still unclear but is believed to involve multivariant genes. The present study showed genetic heterogeneity of stem cell marker in clinically diagnosed NTDs. The variation in stem cell gene mutation is due to the following 4 reasons that could not be ruled out from the present study: a) heterogeneous group of population of NTDs varying in age, gender, and different ethnicity, b) variation in the stem cell gene mutation due to severity of disease recognizing specific region prone for genetic susceptibility, c) cervical region seems to be more prone due to involvement of all the selected stem cell markers, d) anencephaly and encephalocele suggesting fail to maintain autopluripotency due to sequential downregulation of Oct4, Nanog3, and Sox2 responsible for severity of diseases. Hence, it is hypothesized that genetic diversity of the stem cell markers is associated with the development of NTDs.

\section{ACKNOWLEDGMENTS}

Research supported by the Department of Biotechnology, Ministry of Science \& Technology, Government of India (grant \#BT/PR/9022/MED/12/331/2007). We are grateful to the patients and their family members who participated in this study. 


\section{REFERENCES}

Anonymous (1991). Prevention of neural tube defects: results of the Medical Research Council Vitamin Study. MRC Vitamin Study Research Group. Lancet 338: 131-137.

Abzhanov A, Tzahor E, Lassar AB and Tabin CJ (2003). Dissimilar regulation of cell differentiation in mesencephalic (cranial) and sacral (trunk) neural crest cells in vitro. Development 130: 4567-4579.

Beaudin AE and Stover PJ (2009). Insights into metabolic mechanisms underlying folate-responsive neural tube defects: a minireview. Birth Defects Res. A Clin. Mol. Teratol. 85: 274-284.

Bhatia B, Singhal S, Tadman DN, Khaw PT, et al. (2011). SOX2 is required for adult human muller stem cell survival and maintenance of progenicity in vitro. Invest. Ophthalmol. Vis. Sci. 52: 136-145.

Botto LD and Yang Q (2000). 5,10-Methylenetetrahydrofolate reductase gene variants and congenital anomalies: a HuGE review. Am. J. Epidemiol. 151: 862-877.

Cabrera RM, Hill DS, Etheredge AJ and Finnell RH (2004). Investigations into the etiology of neural tube defects. Birth Defects Res. C. Embryo Today 72: 330-344.

Chambers I and Smith A (2004). Self-renewal of teratocarcinoma and embryonic stem cells. Oncogene 23: 7150-7160.

Couly G, Creuzet S, Bennaceur S, Vincent C, et al. (2002). Interactions between Hox-negative cephalic neural crest cells and the foregut endoderm in patterning the facial skeleton in the vertebrate head. Development 129: 1061-1073.

Deb-Rinker P, Ly D, Jezierski A, Sikorska M, et al. (2005). Sequential DNA methylation of the Nanog and Oct-4 upstream regions in human NT2 cells during neuronal differentiation. J. Biol. Chem. 280: 6257-6260.

Detrait ER, George TM, Etchevers HC, Gilbert JR, et al. (2005). Human neural tube defects: developmental biology, epidemiology, and genetics. Neurotoxicol. Teratol. 27: 515-524.

Fernandes KJ, McKenzie IA, Mill P, Smith KM, et al. (2004). A dermal niche for multipotent adult skin-derived precursor cells. Nat. Cell Biol. 6: 1082-1093.

Finnell RH, Junker WM, Wadman LK and Cabrera RM (2002). Gene expression profiling within the developing neural tube. Neurochem. Res. 27: 1165-1180.

Hart AH, Hartley L, Ibrahim M and Robb L (2004). Identification, cloning and expression analysis of the pluripotency promoting Nanog genes in mouse and human. Dev. Dyn. 230: 187-198.

Henderson JK, Draper JS, Baillie HS, Fishel S, et al. (2002). Preimplantation human embryos and embryonic stem cells show comparable expression of stage-specific embryonic antigens. Stem Cells 20: 329-337.

Hunter AG, Cleveland RH, Blickman JG and Holmes LB (1996). A study of level of lesion, associated malformations and sib occurrence risks in spina bifida. Teratology 54: 213-218.

Kruger GM, Mosher JT, Bixby S, Joseph N, et al. (2002). Neural crest stem cells persist in the adult gut but undergo changes in self-renewal, neuronal subtype potential, and factor responsiveness. Neuron 35: 657-669.

Le Lievre CS and Le Douarin NM (1975). Mesenchymal derivatives of the neural crest: analysis of chimaeric quail and chick embryos. J. Embryol. Exp. Morphol. 34: 125-154.

Levasseur DN, Wang J, Dorschner MO, Stamatoyannopoulos JA, et al. (2008). Oct4 dependence of chromatin structure within the extended Nanog locus in ES cells. Genes Dev. 22: 575-580.

Li HY, Say EH and Zhou XF (2007). Isolation and characterization of neural crest progenitors from adult dorsal root ganglia. Stem Cells 25: 2053-2065.

Mitsui K, Tokuzawa Y, Itoh H, Segawa K, et al. (2003). The homeoprotein Nanog is required for maintenance of pluripotency in mouse epiblast and ES cells. Cell 113: 631-642.

Motohashi T, Aoki H, Chiba K, Yoshimura N, et al. (2007). Multipotent cell fate of neural crest-like cells derived from embryonic stem cells. Stem Cells 25: 402-410.

Nettersheim D, Biermann K, Gillis AJ, Steger K, et al. (2011). NANOG promoter methylation and expression correlation during normal and malignant human germ cell development. Epigenetics 6: 114-122.

Nichols J, Zevnik B, Anastassiadis K, Niwa H, et al. (1998). Formation of pluripotent stem cells in the mammalian embryo depends on the POU transcription factor Oct4. Cell 95: 379-391.

Pardal R, Clarke MF and Morrison SJ (2003). Applying the principles of stem-cell biology to cancer. Nat. Rev. Cancer 3: $895-902$.

Partington MD and McLone DG (1995). Hereditary factors in the etiology of neural tube defects. Results of a survey. Pediatr. Neurosurg. 23: 311-316.

Pesce M and Scholer HR (2001). Oct-4: gatekeeper in the beginnings of mammalian development. Stem Cells 19: 271-278.

Rosner MH, Vigano MA, Ozato K, Timmons PM, et al. (1990). A POU-domain transcription factor in early stem cells and germ cells of the mammalian embryo. Nature 345: 686-692.

Seller MJ (1990). Neural tube defects: are neurulation and canalization forms causally distinct? Am. J. Med. Genet. 35:

Genetics and Molecular Research 12 (3): 2380-2390 (2013)

CFUNPEC-RP www.funpecrp.com.br 
394-396.

Sieber-Blum M, Grim M, Hu YF and Szeder V (2004). Pluripotent neural crest stem cells in the adult hair follicle. Dev. Dyn. 231: 258-269.

Tada T and Tada M (2001). Toti-/pluripotential stem cells and epigenetic modifications. Cell Struct. Funct. 26: 149-160.

Takashima Y, Era T, Nakao K, Kondo S, et al. (2007). Neuroepithelial cells supply an initial transient wave of MSC differentiation. Cell 129: 1377-1388

van der Put NM, Steegers-Theunissen RP, Frosst P, Trijbels FJ, et al. (1995). Mutated methylenetetrahydrofolate reductase as a risk factor for spina bifida. Lancet 346: 1070-1071. 